\title{
Rapid and Precise Analysis of Carbon Dioxide Clumped Isotopic Composition by Tunable Infrared Laser Differential Spectroscopy
}

\author{
Zhennan Wang, David D. Nelson ${ }^{\mathrm{b}}$, David L. Dettman*a, J. Barry McManus ${ }^{\mathrm{b}}$, Jay Quade, \\ Katharine W. Huntington ${ }^{c}$, Andrew J. Schauerc, and Saburo Sakai ${ }^{\mathrm{d}}$ \\ ${ }^{a}$ Department of Geosciences, University of Arizona, Tucson, Arizona 85721, United States \\ ${ }^{\mathrm{b}}$ Aerodyne Research, Inc., Billerica, Massachusetts 01821, United States \\ ${ }^{c}$ Department of Earth and Space Sciences, University of Washington, Seattle, Washington 98195, United States \\ d Institute of Biogeochemistry, Japan Agency for Marine-Earth Science and Technology, Yokosuka, Kaganaga 237 - \\ 0061, Japan
}

\section{Supporting Information}

Figure s1. Determine optimal conditions in terms of TILDAS instrument stability

Figure s2. One clumped isotope reordering case

Table s1. $\Delta_{638 \mathrm{raw}}, \boldsymbol{\delta}_{636 \mathrm{raw}}$ and $\boldsymbol{\delta}_{628 \mathrm{raw}}$ measurement results using TILDAS instrument

A $0.35 \% \mathrm{CO}_{2}$ in $\mathrm{N}_{2}$ was used to test the performance of the instrument and determine the optimal conditions. The measurement precisions for varying total pressures can be seen in Figure s1. Each data point in Figure s1(a) represents the one-second standard error. The pressure for optimal absorption and stability in the TILDAS instrument was found in the 50 to 60 Torr range, and larger sample sizes (i.e., higher sample pressure) did not significantly improve performance. Figure s1(b) demonstrates that the measurement precision in terms of standard error is improved as expected by averaging multiple data points.
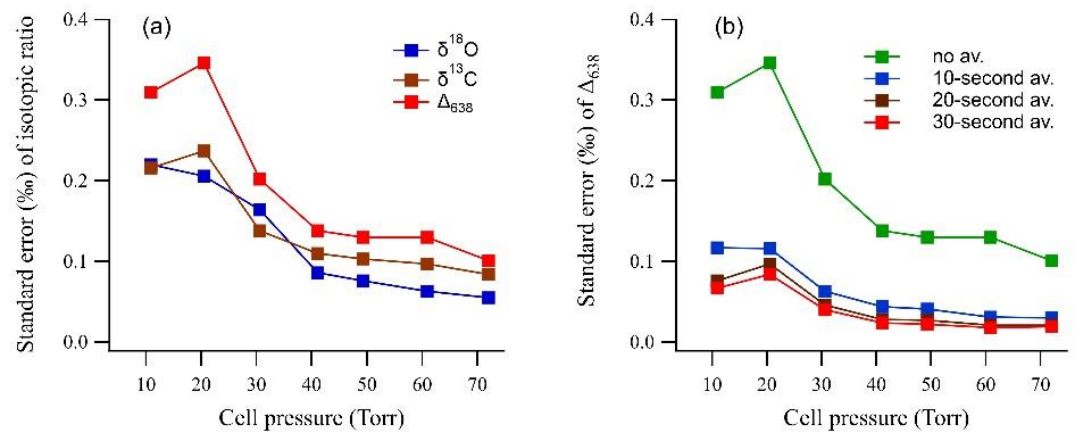

Figure s1. The influence of sample pressure and integration time on TILDAS instrument precision. (a) isotopic ratio measurement precision at various pressures in terms of onesecond standard error. (b) $\Delta_{638}$ measurement precision at various pressures in terms of nsecond average. 
One Clumped Isotope Reordering Case. During testing of a heated $\mathrm{CO}_{2}$ gas sample, we were able to document and follow the active re-ordering of the $\mathrm{CO}_{2}$ isotopologues due to trace water contamination in the sample. To mix the $\mathrm{CO}_{2}$ sample with nitrogen to a concentration of $0.35 \%$, a tube of $\mathrm{CO}_{2}$ was mounted on a tube cracker that was connected to a stainless-steel canister, and a dry scroll vacuum pump with an ultimate pressure of 0.007 Torr was also connected to the canister to pump away unwanted gases. $\mathrm{CO}_{2}-\mathrm{H}_{2} \mathrm{O}$ re-equilibration will occur in a sample when small amounts of water are present, through the $\mathrm{CO}_{2}+\mathrm{H}_{2} \mathrm{O} \leftrightarrows \mathrm{H}_{2} \mathrm{CO}_{3}$ reaction. Out of thirty samples tested during the period of this study, reordering was observed in only one heated sample (Figure s2). Each data point in Figure s2 represents one TILDAS reported $\Delta_{638 \text { raw }}$ or $\delta_{628 \text { raw }}$ value obtained within one subcycle measurement of $5 \mathrm{~min}$. It shows a clear increasing trend of approximately $0.05 \% \mathrm{~h} / \mathrm{h}$ in $\Delta_{638 \mathrm{raw}}$ values, whereas the $\delta_{628 \mathrm{raw}}$ values were relatively stable exhibiting non-monotonic variation. This behavior suggests that trace water was present, leading to progressive $\mathrm{CO}_{2}-\mathrm{H}_{2} \mathrm{O}$ re-equilibration, and that it occurred in the canister holding the sample (due to water absorbed on the canister wall) rather than in the sampling system and optical sample cell. The latter two are unlikely because they were pumped down to vacuum and flushed with nitrogen after each sample/working reference was measured.


Figure s2. (a): the observed clumped isotope reordering behavior in terms of TILDAS reported $\Delta_{638 \text { raw }}$ values in a heated sample and Allan variance plot. (b): the TILDAS reported $\delta_{628 \text { raw }}$ values of the same gas and Allan variance plot. 
Table s1 is the summary of eight equilibrated/heated $\mathrm{CO}_{2}$ sample gases. Each sample was measured twice (i.e., Test 1 and Test 2 in Table s1) with an interval of $8 \mathrm{~h}$ during which the sample remained in the canister. The results show that Test 1 agrees with Test 2 in $\Delta_{638 \text { raw }}$ values with a high internal precision $(1 \mathrm{SE}<0.01 \%$ o).

Table s1. $\Delta_{638 \mathrm{raw}}, \boldsymbol{\delta}_{636 \mathrm{raw}}$ and $\boldsymbol{\delta}_{628 \mathrm{raw}}$ Measurement Results using TILDAS Instrument.

\begin{tabular}{|c|c|c|c|c|c|c|c|c|}
\hline & \multirow{2}{*}{ Sample } & \multicolumn{4}{|c|}{$\Delta_{638 \mathrm{raw}}(\% \mathrm{o})$} & \multirow{2}{*}{$\mathrm{m}$} & \multirow{2}{*}{$\begin{array}{c}\delta_{636 \mathrm{raw}}(\%) \\
\text { Mean } \pm 1 \mathrm{SD}\end{array}$} & \multirow{2}{*}{$\begin{array}{c}\delta_{628 \text { raw }}(\%) \\
\text { Mean } \pm 1 \text { SD }\end{array}$} \\
\hline & & $\mathrm{n}$ & Mean & $1 \mathrm{SD}$ & $1 \mathrm{SE}$ & & & \\
\hline \multirow{8}{*}{ test 1} & FC4C & 3 & 0.201 & 0.010 & 0.006 & 12 & $26.936 \pm 0.013$ & $59.439 \pm 0.015$ \\
\hline & FF4C & 5 & 0.225 & 0.013 & 0.006 & 20 & $1.934 \pm 0.016$ & $-17.763 \pm 0.013$ \\
\hline & FC22C & 4 & 0.098 & 0.013 & 0.007 & 16 & $26.911 \pm 0.020$ & $55.968 \pm 0.015$ \\
\hline & FF22C & 5 & 0.075 & 0.011 & 0.005 & 21 & $1.904 \pm 0.017$ & $-20.916 \pm 0.011$ \\
\hline & FC60C & 6 & -0.080 & 0.021 & 0.009 & 20 & $26.887 \pm 0.022$ & $48.849 \pm 0.010$ \\
\hline & FF60C & 3 & -0.070 & 0.011 & 0.007 & 12 & $1.890 \pm 0.018$ & $-27.387 \pm 0.012$ \\
\hline & FC1000C & 8 & -0.867 & 0.014 & 0.005 & 34 & $26.878 \pm 0.015$ & $19.102 \pm 0.020$ \\
\hline & FF1000C & 9 & -0.906 & 0.012 & 0.004 & 36 & $1.881 \pm 0.015$ & $0.741 \pm 0.017$ \\
\hline \multirow{8}{*}{ test 2} & FC4C & 3 & 0.211 & 0.015 & 0.009 & 12 & $26.911 \pm 0.016$ & $59.458 \pm 0.013$ \\
\hline & FF4C & 5 & 0.252 & 0.020 & 0.009 & 20 & $1.923 \pm 0.016$ & $-17.777 \pm 0.009$ \\
\hline & FC22C & 4 & 0.123 & 0.014 & 0.007 & 19 & $26.899 \pm 0.019$ & $56.003 \pm 0.024$ \\
\hline & FF22C & 6 & 0.102 & 0.014 & 0.005 & 25 & $1.894 \pm 0.043$ & $-20.944 \pm 0.020$ \\
\hline & FC60C & 3 & -0.075 & 0.010 & 0.006 & 12 & $26.872 \pm 0.022$ & $48.847 \pm 0.055$ \\
\hline & FF60C & 3 & -0.073 & 0.024 & 0.014 & 12 & $1.859 \pm 0.016$ & $-27.398 \pm 0.116$ \\
\hline & FC1000C & 15 & -0.866 & 0.024 & 0.006 & 63 & $26.880 \pm 0.023$ & $19.111 \pm 0.012$ \\
\hline & FF1000C & 11 & -0.904 & 0.014 & 0.004 & 47 & $1.874 \pm 0.017$ & $0.692 \pm 0.026$ \\
\hline
\end{tabular}

$\mathrm{n}$ : number of analysis, each analysis is comprised of four working reference-sample measurement subcycles. m: number of measurement subcycle. FC: fermented corn. FF: fossil fuel. SD: standard deviation. SE: standard error. 